\title{
EARLY-PHASE PHOTOMETRY AND SPECTROSCOPY OF TRANSITIONAL TYPE la SN 2012ht: DIRECT CONSTRAINT ON THE RISE TIME
}

\section{AUTHOR(S):}

Yamanaka, Masayuki; Maeda, Keiichi; Kawabata, Miho; Tanaka, Masaomi; Takaki, Katsutoshi; Ueno, Issei;

Masumoto, Kazunari; ... Matsumoto, Katsura; Nogami, Daisaku; Yoshida, Michitoshi

\section{CITATION:}

Yamanaka, Masayuki ...[et al]. EARLY-PHASE PHOTOMETRY AND SPECTROSCOPY OF TRANSITIONAL TYPE la SN 2012ht: DIRECT CONSTRAINT ON THE RISE TIME. The Astrophysical Journal 2014, 782(2): L35.

\section{ISSUE DATE:}

2014-02-20

URL:

http://hdl.handle.net/2433/182939

\section{RIGHT:}

(c) 2014. The American Astronomical Society. 


\title{
EARLY-PHASE PHOTOMETRY AND SPECTROSCOPY OF TRANSITIONAL TYPE Ia SN 2012ht: DIRECT CONSTRAINT ON THE RISE TIME
}

\author{
Masayuki Yamanaka $^{1}$, Keilchi Maeda ${ }^{2}$, Mino Kawabata ${ }^{3}$, Masaomi Tanaka ${ }^{4}$, Katsutoshi Takaki ${ }^{5}$, \\ Issei Ueno ${ }^{5}$, KaZunari Masumoto ${ }^{3}$, KoJI S. Kawabata ${ }^{6}$, Ryosuke ItoH $^{5}$, Yuki Moritani ${ }^{6}$, \\ Hiroshi Akitaya ${ }^{6}$, Akira Arai ${ }^{7}$, Satoshi Honda $^{7}$, Koichi Nishiyama ${ }^{8}$, Fujio Kabashima ${ }^{9}$, \\ Katsura Matsumoto $^{3}$, Daisaku Nogami ${ }^{1}$, and Michitoshi Yoshida ${ }^{6}$ \\ ${ }^{1}$ Kwasan Observatory, Kyoto University, 17-1 Kitakazan-ohmine-cho, Yamashina-ku, \\ Kyoto 607-8471, Japan; yamanaka@kwasan.kyoto-u.ac.jp \\ 2 Department of Astronomy, Kyoto University, Kitashirakawa-Oiwake-cho, Sakyo-ku, Kyoto 606-8502, Japan \\ ${ }^{3}$ Astronomical Institute, Osaka Kyoiku University, Asahigaoka, Kashiwara, Osaka 582-8582, Japan \\ ${ }^{4}$ National Astronomical Observatory of Japan, Osawa, Mitaka, Tokyo 181-8588, Japan \\ ${ }^{5}$ Department of Physical Science, Hiroshima University, Kagamiyama 1-3-1, Higashi-Hiroshima 739-8526, Japan \\ ${ }^{6}$ Hiroshima Astrophysical Science Center, Hiroshima University, Higashi-Hiroshima, Hiroshima 739-8526, Japan \\ ${ }^{7}$ Center for Astronomy, University of Hyogo, 407-2 Nishigaichi, Sayo-cho, Sayo, Hyogo 679-5313, Japan \\ ${ }^{8}$ Kurume, Fukuoka-ken, Japan \\ ${ }^{9}$ Miyaki-cho, Saga-ken, Japan \\ Received 2013 November 11; accepted 2014 January 19; published 2014 February 10
}

\begin{abstract}
We report photometric and spectroscopic observations of the nearby Type Ia Supernova (SN Ia) 2012ht from -15.8 days to +49.1 days after $B$-band maximum. The decline rate of the light curve is $\Delta m_{15}(B)=1.39 \pm 0.05 \mathrm{mag}$, which is intermediate between normal and subluminous SNe Ia, and similar to that of the "transitional" Type Ia SN 2004eo. The spectral line profiles also closely resemble those of SN 2004eo. We were able to observe SN 2012ht at a very early phase, when it was still rising and was about three magnitudes fainter than at the peak. The rise time to the $B$-band maximum is estimated to be $17.6 \pm 0.5$ days and the time of the explosion is MJD 56277.98 \pm 0.13 . SN 2012ht is the first transitional SN Ia whose rise time is directly measured without using light curve templates, and the fifth SN Ia overall. This rise time is consistent with those of the other four SNe within the measurement error, even including the extremely early detection of SN 2013dy. The rising part of the light curve can be fitted by a quadratic function, and shows no sign of a shock-heating component due to the interaction of the ejecta with a companion star. The rise time is significantly longer than that inferred for subluminous SNe such as SN 1991bg, which suggests that a progenitor and/or explosion mechanism of transitional SNe Ia are more similar to normal $\mathrm{SNe}$ Ia rather than to subluminous SNe Ia.
\end{abstract}

Key words: supernovae: general - supernovae: individual (SN 2012ht, SNe 2009ig, 2011fe, 2012cg, 2004eo)

Online-only material: color figures

\section{INTRODUCTION}

When the mass of a carbon-oxygen white dwarf approaches the Chandrasekhar limiting mass, thermonuclear runaway starts, and the whole star explodes. This is thought to be the origin of Type Ia supernova (SN Ia). Since the explosion occurs with this limiting mass, observational properties of SNe Ia are homogeneous. The peak luminosity is correlated with the decline rate of the light curves (Phillips 1993). Using these properties, the acceleration of the cosmic expansion was discovered (Perlmutter et al. 1999; Riess et al. 1998). In spite of the importance of SNe Ia in the field of astrophysics and cosmology, the progenitor scenario and explosion mechanism are not yet well understood (Hillebrandt \& Niemeyer 2000).

The rising part of SNe Ia can provide important constraints on the progenitor system (e.g., Kasen 2010) and the explosion mechanism (e.g., Piro et al. 2010). Recently, detailed studies on the very early phases have been reported for several objects, which include SNe 2009ig (Foley et al. 2012; Marion et al. 2013), 2011fe (Nugent et al. 2011; Bloom et al. 2012; Vinkó et al. 2012; Pereira et al. 2013), 2012cg (Silverman et al. 2012; Munari et al. 2013), and 2013dy (Zheng et al. 2013). These SNe are thought to be discovered within 2 days after the explosion, at 4 mag fainter than its peak. The best case is SN $2011 \mathrm{fe}$. The rising part of the light curve was well fitted by a quadratic function from 0.2 days after the explosion time (Bloom et al. 2012). Detailed study of the rising part of the light curves is still limited to a small number of objects.

In this Letter, we present another excellent observational example of a SN Ia at a very early phase. We discovered SN 2012ht on 2012 December 18.772 UT at an unfiltered magnitude of $17.15 \pm 0.09 \mathrm{mag}^{10}$ in the nearby galaxy NGC 3447 (Nishiyama et al. 2012), whose redshift is 0.003559 (NASA Extragalactic Database, NED). An additional observation was performed, showing that the object brightened to an unfiltered magnitude of $16.23 \pm 0.08 \mathrm{mag}$ on December 19.72. On the other hand, this SN was not detected ( $\geqslant 18.6 \mathrm{mag}$ ) on December 16.7362 .0 days before the discovery (Nishiyama et al. 2012). Spectroscopic observations soon confirmed that it was a young SN Ia (Milisavljevic \& Martin 2012; Yamanaka et al. 2012). We performed photometric and spectroscopic observations of SN 2012 ht from -15.8 days to +49.1 days after the $B$-band maximum. As shown below, we determine that the epoch of the discovery is just 1.8 days after the explosion. We classify this $\mathrm{SN}$ as a transitional SN Ia between normal and subluminous $\mathrm{SNe}$ Ia, based on the light curves and spectral line profiles. The rise time of SN 2012ht is compared with those of a few other $\mathrm{SNe}$ Ia of which the rise time have been accurately estimated.

\footnotetext{
10 The magnitudes on December 18.772 and December 19.72 were
} recalibrated (see Section 2). 

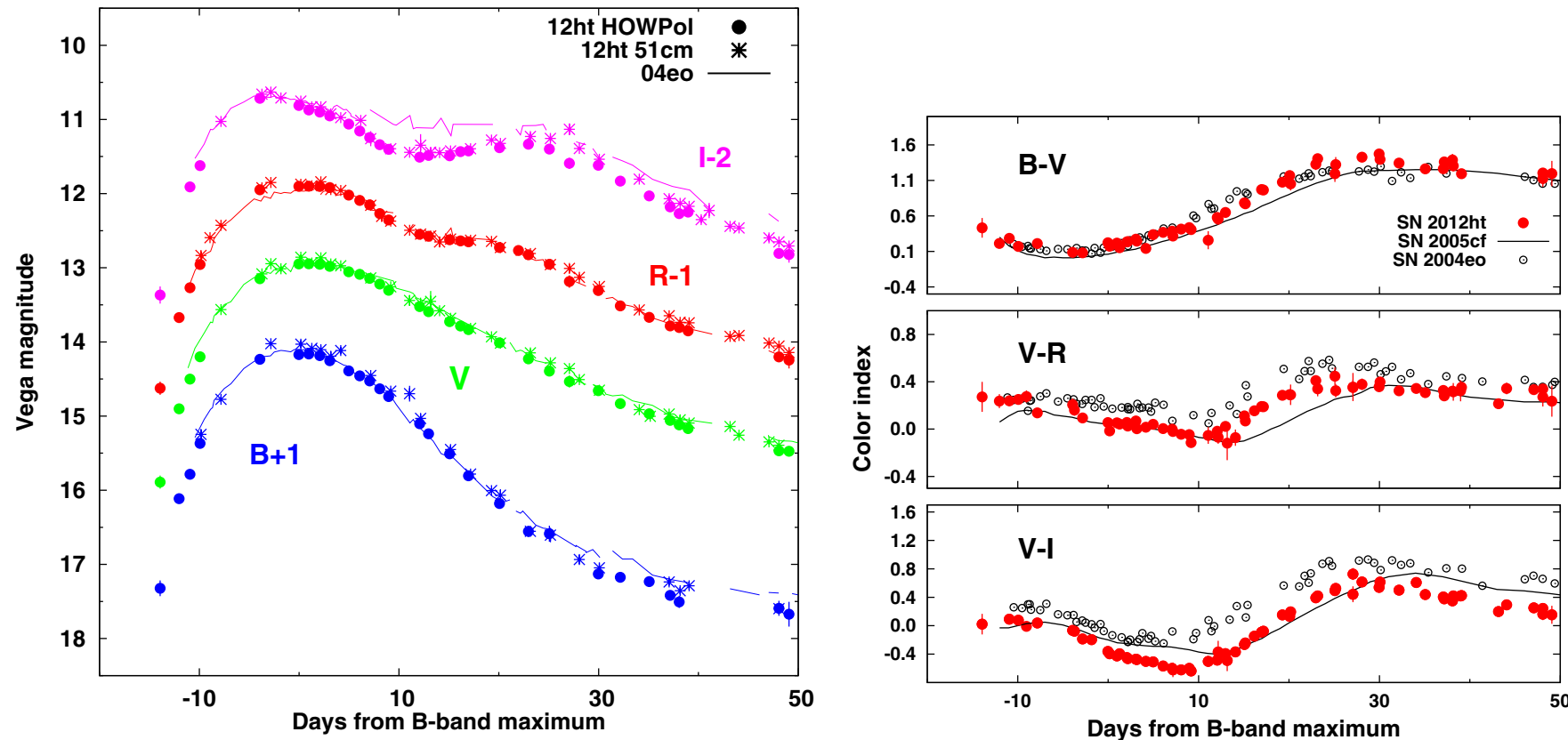

Figure 1. Left panel: $B-, V-, R$-, and $I$-band light curves of SN 2012ht compared with those of another transitional SN Ia, 2004eo (Pastorello et al. 2007). Galactic extinction has been corrected for using the values from Schlafly \& Finkbeiner (2011). The filled and open circles denote the data obtained by the $1.5 \mathrm{~m}$ Kanata and $51 \mathrm{~cm}$ telescopes, respectively. The lines denote the light curves of SN 2004eo. The $B$-band maximum date and magnitude are determined to be MJD $56295.60 \pm 0.61$ and $13.22 \pm 0.04$ mag by polynomial fitting. The maximum magnitudes of SN 2004eo are shifted to match those of SN 2012ht for comparison. Right panel: $B-V$, $V-R$ and $V-I$ color evolution compared to those of SN 2004eo (Pastorello et al. 2007) and a typical SN Ia 2005cf (Wang et al. 2009). The error bar of each data point denotes the square root of the sum of observational error $(1 \sigma)$ and the systematic error of our photometry.

(A color version of this figure is available in the online journal.)

We also discuss the absence of excess emission in the earliest phases and give an implication for a progenitor scenario and explosion mechanism.

\section{OBSERVATIONS AND DATA REDUCTION}

We performed $B-, V-, R$-, and $I$-band imaging observations of SN 2012ht on 33 nights from 2012 December 20.7 UT ( -13.9 days) to 2013 February 21.7 (+49.1 days) using Hiroshima One-shot Wide-field Polarimeter (HOWPol; Kawabata et al. 2008) mounted on the $1.5 \mathrm{~m}$ Kanata telescope at Higashi-Hiroshima Observatory, and on 40 nights from -10.5 to +49.1 days using the $51 \mathrm{~cm}$ telescope in Osaka Kyoiku University. The images were reduced according to standard procedures of CCD photometry. We performed aperture photometry on HOWPol data and point-spread function (PSF) fitting photometry on the $51 \mathrm{~cm}$ telescope's data using IRAF. The magnitude is calibrated with photometric standard stars in Landolt fields observed on photometric nights. We corrected the magnitudes obtained by these two instruments using their color terms. Color terms were derived through the observations of the standard field M67 (Chevalier \& Ilovaisky 1991) on a photometric night. The magnitudes obtained with these instruments are consistent within a systematic error of 0.1-0.2 mag at most. We also re-reduced the unfiltered discovery image obtained by Nishiyama et al. (2012) using PSF photometry. The same applies to the additional image on December 19.7. The magnitudes are calibrated using local standard stars whose $R$ magnitudes are estimated by HOWPol.

For spectroscopy, we obtained optical spectra using HOWPol on 12 nights from -13.9 to +7.1 days and also using MALLS installed on the $2.0 \mathrm{~m}$ Nayuta telescope at the Nishi-Harima Astronomical Observatory (NHAO) on four nights from -9.9 to +7.9 days. Their wavelength coverage and spectral resolutions are 4500-9000 $\AA$ and $R=\lambda / \Delta \lambda=400$ (at $6000 \AA$ ) for HOWPol and 4200-6800 and $R=1200$ (at $6000 \AA$ ) for MALLS. For the MALLS data on December 26, they are instead 4200-9000 and $R=500$ (at $6000 \AA$ ). We performed wavelength calibration using telluric emission lines of the sky superimposed on each object frame for the HOWPol data and using comparison lamp ( $\mathrm{FeArNe}$ ) frames for the MALLS data. The fluxes have been calibrated using spectrophotometric standard star data.

\section{RESULTS}

\subsection{Light Curves and Color}

The $B-, V-, R$-, and $I$-band light curves of SN $2012 \mathrm{ht}$ are shown in Figure 1. These data have been corrected for the Galactic extinction of $E(B-V)=0.02 \mathrm{mag}$ and $R_{V}=3.1$ (Schlafly \& Finkbeiner 2011). The extinction within the host galaxy is assumed to be negligible as discussed in the next paragraph. The $B$-band maximum date and magnitude are derived to be MJD $56295.60 \pm 0.61$ (2013 January 3.6 UT) and $13.22 \pm 0.04 \mathrm{mag}$, respectively, by polynomial fitting at around maximum. Throughout this Letter, the phase $t$ is expressed relative to the $B$-band maximum date, i.e., $t=0$ is MJD 56295.60.

We also use this definition in discussing the rise time in Section 3.2. The light curves of SN 2012ht are similar to those of SN 2004eo $\left(\Delta m_{15}(B)=1.46 \mathrm{mag}\right.$; Pastorello et al. 2007) in all bands. SN 2004eo is classified as a transitional SN Ia, which is intermediate between normal and subluminous SNe Ia (Pastorello et al. 2007). We derived the decline-rate parameter of SN2012ht as $\Delta m_{15}(B)=1.39 \pm 0.05 \mathrm{mag}$, which is close to that of SN 2004eo. The time of the $I$ secondary maximum is at $t=22.5 \pm 0.5$ days, which is also consistent with that of 
SN 2004eo. A small difference can be seen only in the I-band magnitude after the first maximum; SN 2012ht shows a clearer dip between the first and secondary maximum than SN 2004eo. We confirm the 3.2, 2.9, 2.7, and 2.6 mag brightening in the $B, V, R$ and $I$ bands, respectively, from $t=-13.9$ to $B$-band maximum. It is remarkable that we were able to perform multiband photometry at such early phases, thanks to the follow-up observations from a very early phase.

The distance modulus for NGC 3447 has a relatively large uncertainty due to its infall velocity to the Virgo cluster (Marino et al. 2010). Adopting the Tully-Fisher distance of $\mu=31.5 \pm 0.4$ from NED, the absolute magnitude of SN $2012 \mathrm{ht}$ is calculated to be $M_{B}=-18.3 \pm 0.4 \mathrm{mag}$. It is noted that its magnitude is fainter than the $M_{B}=-19.14 \pm 0.10$ mag expected from its decline-rate parameter of $\Delta m_{15}(B)=1.39 \mathrm{mag}$ (Prieto et al. 2006).

The color evolution of SN 2012ht compared to the intrinsic color (i.e., extinction-corrected color) evolution of SNe 2005cf (Wang et al. 2009) and 2004eo (Pastorello et al. 2007) is shown in the right panel of Figure 1 . The $B-V$ color of SN 2012ht is comparable to that of SNe 2005cf and 2004eo around maximum. This overall similarity of the color evolution suggests that the extinction in the host galaxy of SN 2012ht is negligible based on the Lira relation (Lira et al. 1998). The color evolution in different bandpasses is similar to SN 2004eo, which is of the same subtype as SN 2012ht, except bluer in $V-R$ and $V-I$. This might be a characteristic of SNe Ia with detected carbon absorption (e.g., Thomas et al. 2011).

\subsection{Spectral Properties}

We show the spectral evolution of SN Ia 2012ht from $t=-13.9$ to +7.1 days in Figure 2. Spectra have been corrected for the recession velocity of the host galaxy NGC 3447 from NED. The atmospheric absorption lines were removed using standard star data obtained at the same nights except for the spectrum at $t=-13.9$ days.

The O I $\lambda 7774$ absorption line in SN 2012ht is very strong compared with other SNe Ia at similar epochs as shown in the lower two panels in Figure 2. The equivalent width is measured to be $105 \AA$ using the spectrum at $t=+0.1$ days. Hachinger et al. (2006) investigate the relationship of the equivalent widths of several absorption lines around maximum with the decline-rate $\Delta m_{15}(B)$ in SNe Ia. The equivalent width of the O I $\lambda 7774$ absorption line implies $\Delta m_{15}(B)=1.4-1.8 \mathrm{mag}$. This is consistent with the intermediate decline rate of $\Delta m_{15}(B)=$ $1.4 \mathrm{mag}$ found for SN 2012ht. The line-depth ratio ( $R$ (Si II)) of Si II $\lambda 5972$ to $\mathrm{Si}$ II $\lambda 6355$ is an indicator of the absolute magnitude of SNe Ia (Nugent et al. 1995). We measured the ratio to be $R(\mathrm{Si}$ II $) \sim 0.34$ using the spectrum at $t=+0.1$ days. This value is also consistent with that expected from the relation between $\Delta m_{15}(B)$ and $R(\mathrm{Si}$ II) (Figure 14 of Blondin et al. 2012). This indicates that SN 2012ht is located in the faint end of normal SNe Ia. At 4500-5100 A, the broad features are blends of Fe II multiplet (4923, 5018, 5169), Fe III, and Si II $\lambda 5056$ absorption lines, which are seen in normal and overluminous SNe Ia (Branch et al. 2006). For SN 2012ht, the absorption line at $4850 \AA$ is very strong after $t=-11.9$ days. It is very similar to the transitional SN Ia 2004eo (see the lower two panels in Figure 2 and Pastorello et al. 2007). In transitional SNe Ia 1986G, 1992A, and 2009an, this absorption line is also clearly stronger than those of normal SNe Ia (Phillips et al. 1987; Kirshner et al. 1993; Sahu et al. 2013). These spectral properties support the fact that SN 2012ht, like SN 2004eo, belongs to the transitional group.

The shallow $C_{\text {II }} \lambda 6580$ absorption line is seen around $6300 \AA$ in spectra until -8.9 days, and it disappears by -3.9 days. We attempt to seek C II $\lambda 7234$ in our spectra. However, it was not significantly detected in our spectra. The line velocity of C II in SN 2012ht is measured to be $12,000 \mathrm{~km} \mathrm{~s}^{-1}$, which is the same as that of S II $\lambda 6355$ within the measurement error at $t=-11.9$ days. On the other hand, $\mathrm{C}_{\mathrm{II}}$ absorption lines are not seen in SN 2004eo (Pastorello et al. 2007).

\section{DISCUSSION}

\subsection{Estimate of the Rise Time}

SN 2012ht is the first transitional SN Ia for which the rise time can be accurately estimated by early-phase observations. To give a direct constraint on the rise time from observational data, we attempt to fit a quadratic curve to the rising part of the $R$-band light curve of SN 2012ht (see right panel of Figure 3 ). We find that the rising curve up to $t=-8$ days is well fitted within the photometric error. The explosion time was determined to be MJD $56277.98 \pm 0.13$ by extending the fitting curve to the zero-point flux level. Surprisingly, the discovery date by K. Nishiyama and F. Kabashima was 1.8 days after this explosion time (Nishiyama et al. 2012), and the upper-limit by K. Itagaki (Nishiyama et al. 2012) was obtained 0.2 days before the explosion.

We estimate the rise time of SN 2012ht to be $17.62 \pm$ 0.52 days, ${ }^{11}$ which is compared to that of $\mathrm{SNe} 2004 \mathrm{eo}$ (Pastorello et al. 2007), 2009ig (Foley et al. 2012), 2011fe (Vinkó et al. 2012), and 2012cg (Munari et al. 2013) in the left panel of Figure 3. The maximum magnitude is shifted to zero for all objects. The time is referred to the $B$-band maximum date for each event. The rising rate of SN 2012ht is similar to those of SNe 2009ig and 2011fe (Figure 3) despite a variety of luminosity classes covered by these objects (see also Figure 4). SN $2011 \mathrm{fe}$ is classified as a very normal SN Ia based on photometric and spectroscopic observations (Vinkó et al. 2012; Pereira et al. 2013). On the other hand, SN 2009ig shows an extremely slow decline rate in the $B$-band light curve despite having typical absolute magnitude (Foley et al. 2012; Marion et al. 2013).

We then compare the rise time of SN 2012ht with those of underluminous objects. Lacking a direct constraint on the rise time of these faint SNe Ia, we rely on statistical studies (Riess et al. 1999; Hayden et al. 2010; Ganeshalingam et al. 2011; González-Gaitán et al. 2012). To estimate the rise time, they used a template light curve which is constructed from large samples. We present the rise time and the decline rate in the $B$ band from Ganeshalingam et al. (2011) compared to those of SNe 2009ig, 2011fe, 2012cg, 2013dy, and 2012ht in Figure 4. Underluminous objects which are spectroscopically classified as SN 1991bg-like (Filippenko et al. 1992) have relatively short rise times of 13-15 days (Modjaz et al. 2001; Taubenberger et al. 2008; Ganeshalingam et al. 2011). Figure 4 shows that the rise time of SN 2012ht is significantly larger than many of these underluminous events, but is similar to that of normal and overluminous $\mathrm{SNe}$. This implies that the transitional objects can originate from the same explosion mechanism, progenitor, and/or population, as normal and overluminous events.

\footnotetext{
11 The rise time is defined as the $B$-band maximum date minus the explosion date, the same definition used in previous studies (Foley et al. 2012; Nugent et al. 2011; Silverman et al. 2012).
} 


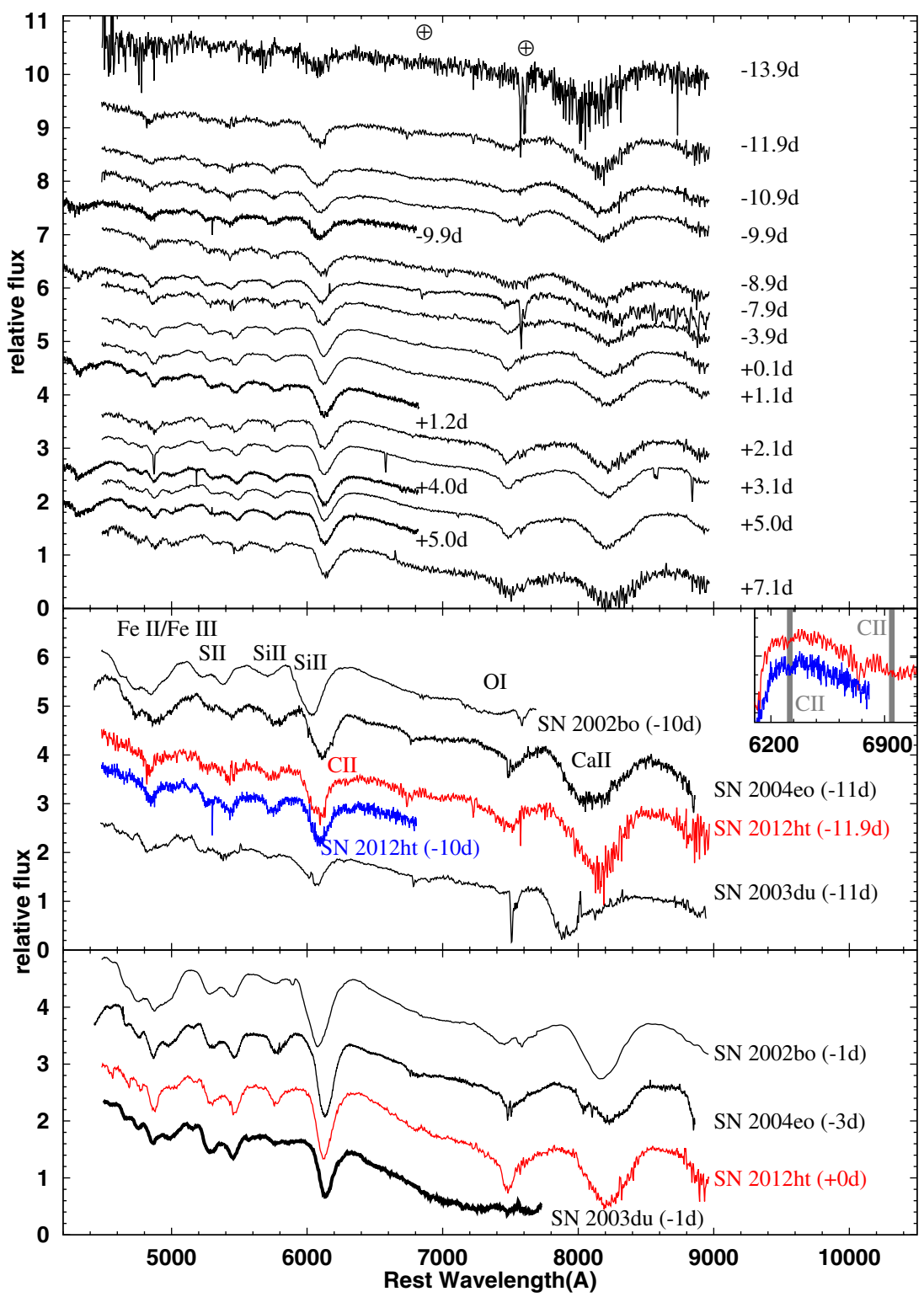

Figure 2. Upper panel: time evolution of spectra of SN 2012ht. These spectra are corrected for its recession velocity. Telluric absorption lines remain in the spectrum at -13.9 days due to a low $\mathrm{S} / \mathrm{N}$ ratio. The phases refer to the $B$-band maximum dates at MJD $56295.60 \pm 0.61$. Middle panel: spectra at $t=-10$ and -12 days compared with those of SNe 2002bo (Benetti et al. 2004), 2003du (Stanishev et al. 2007), and 2004eo (Pastorello et al. 2007) at similar phases. The redshifts of host galaxies were corrected for. The blue and red lines denote spectra obtained by the Nayuta and Kanata telescopes, respectively. The tiny right top panel exhibits the close-up of the $C_{\text {II }} \lambda \lambda 6580,7234$ absorption lines. The two vertical lines denote the blueshift by $12,000 \mathrm{~km} \mathrm{~s}^{-1}$ of these absorption lines. Bottom panel: same as the middle panel at $t=0$.

(A color version of this figure is available in the online journal.)

\subsection{Progenitors and Explosions}

In this section, we discuss the implications of our data for a progenitor system and explosion mechanism of SN 2012ht. The shock luminosity is predicted to be seen at an early phase in several models (Kasen 2010; Piro et al. 2010; Piro \& Nakar 2013; Rabinak \& Waxman 2011). Shock luminosity could be determined by a progenitor radius, an ejected mass, and opacity (Piro et al. 2010; Rabinak \& Waxman 2011). We assume that the ejecta mass and opacity of SN 2012ht are not significantly different from those of SN 2011fe. Additionally, shock luminosity exhibits the power-law decay with time evolution. Bloom et al. (2012) gave the constraint on a progenitor radius of SN $2011 \mathrm{fe}$ as $\lesssim 0.02 R_{\odot}$ using an analytic model (Kasen 2010; Piro et al. 2010; Rabinak \& Waxman 2011) of the emission at $t=0.2$. To estimate the progenitor radius of SN 2012ht, we scaled the parameters from SN 2011fe (Bloom et al. 2012) in the three cases from Kasen (2010), Piro et al. (2010), and Rabinak \& Waxman (2011). We calculate the putative shock luminosity of SN 2011fe at $t=1.8$ days using the radius ( $\sim 0.02 R_{\odot}$; Bloom et al. 2012$)$ in each model (Kasen 2010; Piro et al. 2010; Rabinak \& Waxman 2011). The radius of SN $2012 \mathrm{ht}$ is estimated by scaling this luminosity. We give an upper limit on the radius of $1.5-2.7 R_{\odot}$, which indicates that the progenitor was a (relatively) compact object.

Kasen (2010) suggested that a strong emission may come from the interaction of the ejecta with its companion star. For a $2 M_{\odot}$ red giant $(\mathrm{RG})$ companion star, the shock-induced emission reaches $5.2 \times 10^{41} \mathrm{erg} \mathrm{s}^{-1}$ at $t \lesssim 5$ days even for the viewing angle opposite to the interacting direction (Kasen 

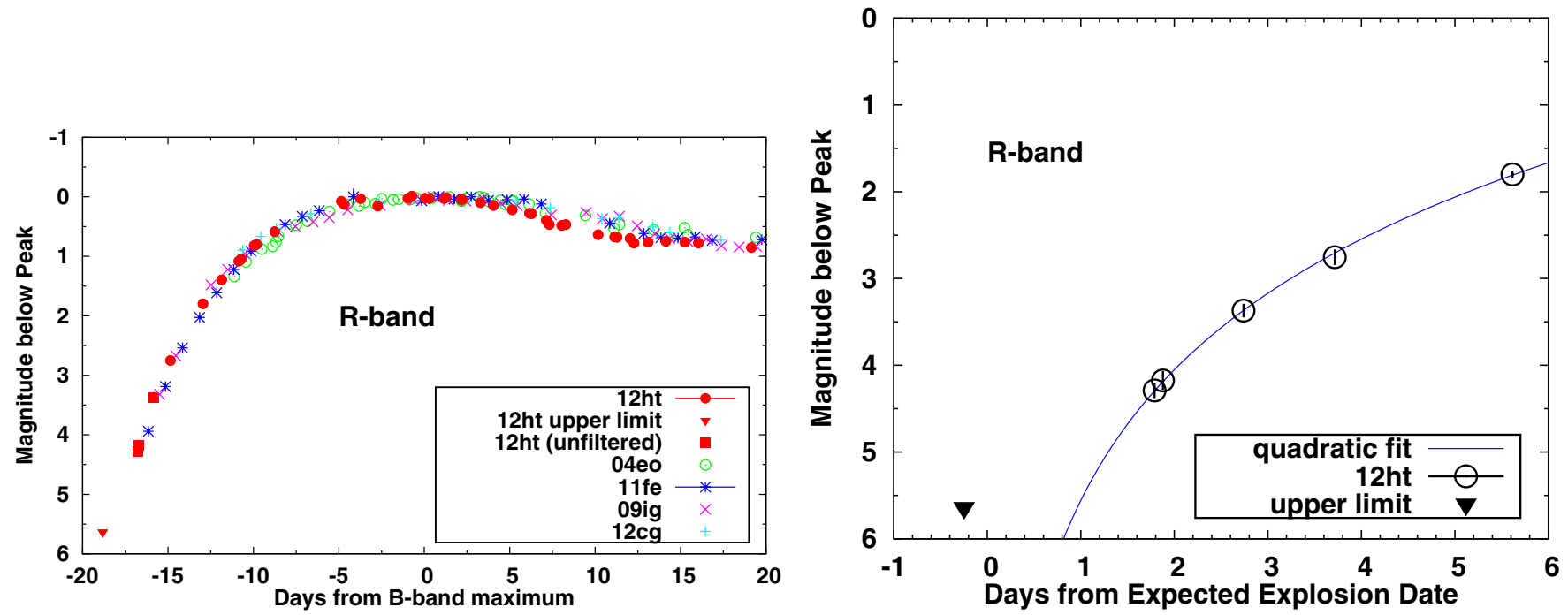

Figure 3. Left panel: early part of the $R$ band of SN 2012ht compared with those of SNe 2004eo (Pastorello et al. 2007), 2009ig (Foley et al. 2012), and 2011fe (Vinkó et al. 2012). The red filled circles denote the data obtained by the $1.5 \mathrm{~m}$ Kanata and $51 \mathrm{~cm}$ telescopes. The red filled squares denote the data obtained by Nishiyama and Kabashima. The upside-down triangle indicates the upper-limit magnitude from K. Itagaki (Nishiyama et al. 2012). The discovery data have been re-calibrated using DAOPHOT. Right panel: close-up of the rising part of the $R$-band light curve from the expected explosion date until 6 days. The explosion date is estimated to be MJD $56277.98 \pm 0.13$ using a fit of a quadratic function denoted by the blue line.

(A color version of this figure is available in the online journal.)

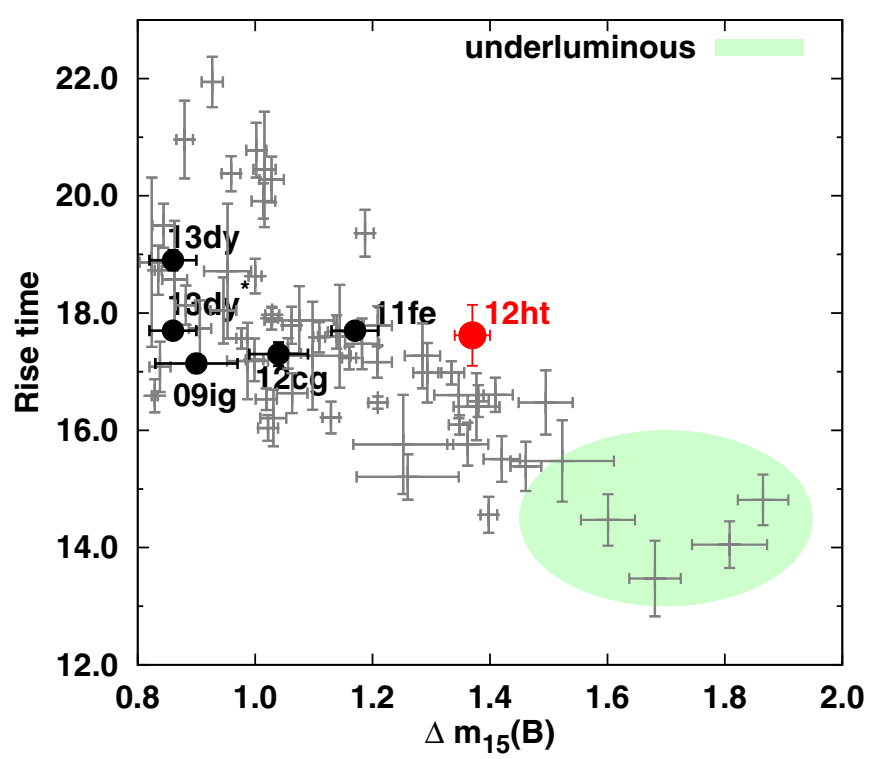

Figure 4. Decline rate $\Delta \mathrm{m}_{15}(B)$ and rise time of SN 2012ht compared with those of SNe 2009ig (Foley et al. 2012; Marion et al. 2013), 2011fe (Nugent et al. 2011; Pereira et al. 2013), 2012cg (Silverman et al. 2012; Munari et al 2013), and 2013dy (Zheng et al. 2013). $\Delta \mathrm{m}_{15}(B)$ of SN 2013dy is $0.86 \pm$ $0.04 \mathrm{mag}$ (W. Zheng 2014, private communication). The rise time of SN 2013dy was calculated to be 17.7 days from the broken power-law fitting, while it is $\sim 18.9$ days when fitting with a quadratic function (Zheng et al. 2013). The gray cross points denote the template-fitting data from Ganeshalingam et al. (2011). The green-shaded area denotes the region that underluminous SNe Ia could occupy.

(A color version of this figure is available in the online journal.)

2010). Since SN $2012 \mathrm{ht}$ is detected at $1.8 \times 10^{41} \mathrm{erg} \mathrm{s}^{-1}$ at $t=1.8$ days, our observations exclude the shock-induced emission by the interaction with an RG companion. It should be noted, however, that we do not reject the possibility that the RG might have already evolved at the time of the explosion through the spin-down scenario (Justham 2011).
If there is a variety in the ${ }^{56} \mathrm{Ni}$ mass distribution within the outer layers of SNe Ia, their rising parts are expected to exhibit some variations (Piro \& Nakar 2012, 2013). Our results indicate that the rising parts of SNe 2012ht, 2011fe, and 2009ig are similar even when transitional SN 2012ht is included (see the left panel of Figure 3). This finding indicates that the distribution of radioactive ${ }^{56} \mathrm{Ni}$ in the outermost layer is similar among these three SNe Ia (see also Piro \& Nakar 2012, 2013). Various models predict different distribution of ${ }^{56} \mathrm{Ni}$ near the surface (e.g., Gamezo et al. 2005; Sim et al. 2010; Seitenzahl et al. 2013), and the "uniformity" we find indicates that these SNe Ia, including transitional and normal SNe Ia, may share a common explosion mechanism.

\section{CONCLUSION}

We successfully performed prompt observations of SN Ia $2012 \mathrm{ht}$. Our data show that SN 2012ht is very similar to a transitional SN 2004eo. The explosion date and the rise time are estimated to be MJD $56277.98 \pm 0.13$ and $17.6 \pm 0.5$ days, respectively. SN 2012ht is the fifth SN Ia for which such an accurate and strong constraint on the rise time has been given directly by observational data. This rise time is comparable to that of normal or overluminous $\mathrm{SNe}$ Ia, but is much longer than that of underluminous SN $1991 \mathrm{bg}$-like objects. This indicates that a common explosion mechanism could be the origin of normal and transitional SNe Ia.

We would like to thank Mohan Ganeshalingam for rise time versus decline rate of light curves data. This research has been supported in part in part by the Optical \& Near-infrared Astronomy Inter-University Cooperation Program (OISTER) and by the Grant-in-Aid for Scientific Research from JSPS (23340048, 24740117, 23244030) and MEXT (25103515, 24103003). Y.M. and R.I. have been supported by the JSPS Research Fellowship for Young Scientists. 


\section{REFERENCES}

Benetti, S., Meikle, P., Stehle, M., et al. 2004, MNRAS, 348, 261 Blondin, S., Matheson, T., Kirshner, R. P., et al. 2012, AJ, 143, 126 Bloom, J. S., Kasen, D., Shen, K. J., et al. 2012, ApJL, 744, L17 Branch, D., Dang, L. C., Hall, N., et al. 2006, PASP, 118, 560 Chevalier, C., \& Ilovaisky, S. A. 1991, A\&AS, 90, 225

Filippenko, A. V., Richmond, M. W., Branch, D., et al. 1992, AJ, 104, 1543 Foley, R. J., Challis, P. J., Filippenko, A. V., et al. 2012, ApJ, 744, 38

Gamezo, V. N., Khokhlov, A. M., \& Oran, E. S. 2005, ApJ, 623, 337 Ganeshalingam, M., Li, W., \& Filippenko, A. V. 2011, MNRAS, 416, 2607 González-Gaitán, S., Conley, A., Bianco, F. B., et al. 2012, ApJ, 745, 44 Hachinger, S., Mazzali, P. A., \& Benetti, S. 2006, MNRAS, 370, 299 Hayden, B. T., Garnavich, P. M., Kasen, D., et al. 2010, ApJ, 722, 1691 Hillebrandt, W., \& Niemeyer, J. C. 2000, ARA\&A, 38, 191 Justham, S. 2011, ApJL, 730, L34

Kasen, D. 2010, ApJ, 708, 1025

Kawabata, K. S., Nagae, O., Chiyonobu, S., et al. 2008, Proc. SPIE, 7014, 70144L

Kirshner, R. P., Jeffery, D. J., Leibundgut, B., et al. 1993, ApJ, 415, 589

Lira, P., Suntzeff, N. B., Phillips, M. M., et al. 1998, AJ, 115, 234

Marino, A., Bianchi, L., Rampazzo, R., Buson, L. M., \& Bettoni, D. 2010, A\&A, 511, A29

Marion, G. H., Vinko, J., Wheeler, J. C., et al. 2013, ApJ, 777, 40

Milisavljevic, D., \& Martin, E. O. 2012, CBET, 3349, 2

Modjaz, M., Li, W., Filippenko, A. V., et al. 2001, PASP, 113, 308

Munari, U., Henden, A., Belligoli, R., et al. 2013, NewA, 20, 30

Nishiyama, K., Kabashima, F., Yusa, T., Itagaki, K., \& Nakano, S. 2012, CBET, 3349,1
Nugent, P., Phillips, M., Baron, E., Branch, D., \& Hauschildt, P. 1995, ApJL, 455, L147

Nugent, P. E., Sullivan, M., Cenko, S. B., et al. 2011, Natur, 480, 344

Pastorello, A., Mazzali, P. A., Pignata, G., et al. 2007, MNRAS, 377, 1531

Pereira, R., Thomas, R. C., Aldering, G., et al. 2013, A\&A, 554, A27

Perlmutter, S., Aldering, G., Goldhaber, G., et al. 1999, ApJ, 517, 565

Phillips, M. M. 1993, ApJL, 413, L105

Phillips, M. M., Phillips, A. C., Heathcote, S. R., et al. 1987, PASP, 99, 592

Piro, A. L., Chang, P., \& Weinberg, N. N. 2010, ApJ, 708, 598

Piro, A. L., \& Nakar, E. 2012, arXiv:1211.6438

Piro, A. L., \& Nakar, E. 2013, ApJ, 769, 67

Prieto, J. L., Rest, A., \& Suntzeff, N. B. 2006, ApJ, 647, 501

Rabinak, I., \& Waxman, E. 2011, ApJ, 728, 63

Riess, A. G., Filippenko, A. V., Challis, P., et al. 1998, AJ, 116, 1009

Riess, A. G., Filippenko, A. V., Li, W., et al. 1999, AJ, 118, 2675

Sahu, D. K., Anupama, G. C., \& Anto, P. 2013, MNRAS, 430, 869

Schlafly, E. F., \& Finkbeiner, D. P. 2011, ApJ, 737, 103

Seitenzahl, I. R., Ciaraldi-Schoolmann, F., Röpke, F. K., et al. 2013, MNRAS, 429,1156

Silverman, J. M., Ganeshalingam, M., Cenko, S. B., et al. 2012, ApJL, 756, L7

Sim, S. A., Röpke, F. K., Hillebrandt, W., et al. 2010, ApJL, 714, L52

Stanishev, V., Goobar, A., Benetti, S., et al. 2007, A\&A, 469, 645

Taubenberger, S., Hachinger, S., Pignata, G., et al. 2008, MNRAS, 385, 75

Thomas, R. C., Aldering, G., Antilogus, P., et al. 2011, ApJ, 743, 27

Vinkó, J., Sárneczky, K., Takáts, K., et al. 2012, A\&A, 546, A12

Wang, X., Li, W., Filippenko, A. V., et al. 2009, ApJ, 697, 380

Yamanaka, M., Takaki, K., Itoh, R., et al. 2012, CBET, 3349, 3

Zheng, W., Silverman, J. M., Filippenko, A. V., et al. 2013, ApJL, 778, L15 